Ruiz-Juan, F.; Baños, R.; Fuentesal-García, J.; García-Montes, E. y Baena-Extremera, A (2019). Análisis transcultural del clima motivacional en alumnado de Costa Rica, México y España / Transcultural Analysis of Motivational Climate in Students in Costa Rica, Mexico and Spain. Revista Internacional de Medicina y Ciencias de la Actividad Física y el Deporte vol. 19 (74) pp. 351-369 Http://cdeporte.rediris.es/revista/revista74/artanalisis1028.htm

DOI: http://doi.org/10.15366/rimcafd2019.74.011

\title{
ORIGINAL
}

\section{ANÁLISIS TRANSCULTURAL DEL CLIMA MOTIVACIONAL EN ALUMNADO DE COSTA RICA, MÉXICO Y ESPAÑA}

\section{TRANSCULTURAL ANALYSIS OF MOTIVATIONAL CLIMATE IN STUDENTS IN COSTA RICA, MEXICO AND SPAIN}

\author{
Ruiz-Juan, F. ${ }^{1}$; Baños, R. ${ }^{2}$; Fuentesal-Garcia, J. ${ }^{3}$; García-Montes, E. ${ }^{1}$ y Baena- \\ Extremera, A. 4
}

${ }^{1}$ Doctor en Ciencias del Deporte. Facultad de Ciencias del Deporte. Universidad de Murcia (España) fruizj@um.es, garciamo@um.es

2 Doctor en Ciencias del Deporte. Facultad de Deportes de Ensenada. Universidad Autónoma de Baja California (México) raulfb89@gmail.com

3 Doctor en Ciencias del Deporte. Centro de Enseñanza Superior Don Bosco. Universidad Complutense de Madrid (España) jfuentesal@cesdonbosco.com

${ }^{4}$ Doctor en Ciencias del Deporte. Facultad de Ciencias de la Educación. Universidad de Granada (España) abaenaextrem@ugr.es

\section{AGRADECIMIENTOS O FINANCIACIÓN}

Este estudio forma parte de un Proyecto longitudinal para medir la influencia de variables de la Educación Física y la actividad física en el tiempo de ocio en los hábitos de práctica a lo largo del tiempo.

Código UNESCO / UNESCO code: 61401 Procesos Congitivos / Cognitive proceses.

Clasificación Consejo de Europa I Classification Council of Europe: 17. Otras: Actividad Física y Salud / Others: Physical Activity and Health.

Recibido 18 de julio de 2017 Received July 18, 2017

Aceptado 15 de enero de 2018 Accepted January 15, 2018

\section{RESUMEN}

El objetivo de este trabajo es conocer como la satisfacción, la motivación y 
las creencias de éxito en las actividades físico-deportivas de tiempo libre del alumnado de educación secundaria, pueden predecir el clima motivacional percibido en Educación Física en tres contextos culturales diferentes. La muestra estuvo compuesta por 2168 estudiantes de tres países diferentes, perteneciendo 423 a Costa Rica, 408 a México y un total de 1337 alumnos a España, todos ellos con edades comprendidas entre 11 y 16 años. Para ello se utilizaron las escalas de Clima Motivacional, Satisfacción Intrínseca en el Deporte, Motivación Deportiva e Inventario de Percepción de las Creencias sobre las Causas del Éxito en el Deporte. Los resultados encontrados en esta investigación muestran similares tendencias en los tres países tanto en la predicción de la maestría, como en el rendimientoaproximación, rendimiento-evitación y en la aprobación social.

PALABRAS CLAVE: Metas de logro, educación física, motivación, actividad física, tiempo libre.

\section{ABSTRACT}

The aim of this paper is to find out how satisfaction, motivation and belief in success in physical and sports leisure time activities of students in secondary education predict the perceived motivational climate in physical education in three different cultural contexts. The sample consisted of 2168 students from three different countries, belonging to Costa Rica 423, 408 to Mexico and a total of 1337 students to Spain, all aged between 11 and 16 years. The Scales Motivational Climate, Satisfaction Intrinsic in Sport, Sport and Inventory Motivation Perceptions of Beliefs on the Causes of Success in Sport were used. The results found in this research show similar trends in all three countries in terms of mastery prediction as well as performance-approach, performance-avoidance, and social approval.

KEYWORDS: Achievement goals, physical education, motivation, physical activity, free time.

\section{INTRODUCCIÓN}

Teniendo como punto de referencia la Teoría de Metas de Logro (Nicholls, 1989) y la Teoría de la Autodeterminación (Deci \& Ryan, 2000), se han elaborado multitud de trabajos de investigación de diversas perspectivas que buscan, entre otros objetivos, explicar conductas y comportamiento del alumnado de Educación Física (EF) (Baños, Ortiz-Camacho, Baena-Extremera, Tristán-Rodríguez, 2017; Gómez-López, Granero-Gallegos, Baena-Extremera, \& Abraldes, 2014; Juliá \& Baena-Extremera, 2018).

Sobre la teoría de Metas de Logro, autores como Papaioannou, Tsigilis, Kosmidou y Milosis (2007) identificaron que los estudiantes se pueden orientar de cuatro formas distintas para la consecución de sus metas: maestría-aproximación, rendimiento-aproximación, rendimiento-evitación y aprobación social. Estas metas, tienen especial importancia en el alumnado por los comportamientos que suponen 
estar más orientado a una meta que otra. Por ejemplo, las metas de maestría según Baena-Extremera y Granero-Gallegos (2015), son predictoras de la satisfacción con la EF y a la escuela, y se relacionan con perfiles de alumnos que buscan mejorar su habilidad, con alta motivación intrínseca, etc. (Roberts \& Treasure, 2018). En cambio, las metas de rendimiento, suelen estar relacionadas con la motivación extrínseca, con un clima de rendimiento y de competencia (Cook, Castillo, Gas, \& Artino, 2017), así como con estados de acercamiento desadaptativo al aprendizaje, desmotivación y ansiedad (Roberts \& Treasure, 2018).

La importancia de estos modelos, radica fundamentalmente por su relación con la motivación en el alumnado de EF. La motivación ha sido estudiada a raíz de varias teorías, siendo una de ellas la nombra Teoría de la Autodeterminación. Siguiendo esta teoría, la motivación puede estar presente de tres formas: intrínseca, extrínseca y amotivación. Ahondando un poco más, la motivación intrínseca presenta hasta tres manifestaciones: la motivación intrínseca por el conocimiento, por el logro y por las experiencias estimulantes (Ryan \& Deci, 2000). En el caso de la extrínseca, relacionada con conductas causadas por razones instrumentales o fuentes externas, siguiendo a Deci y Ryan (2000), se puede distinguir: la regulación identificada, la introyectada y la regulación externa. Finalmente, la amotivación o ausencia relativa de motivación, en la cual el deportista no se encuentra motivado ni intrínseca, ni extrínsecamente (Vella, Swann, Allen, Schweickle, \& Magee, 2017).

La motivación, es una variable que afecta de manera importante al alumno, no sólo en su rendimiento académico, sino incluso en su práctica de actividad física escolar y extraescolar (Granero-Gallegos, Baena-Extremera, Pérez-Quero, OrtizCamacho, \& Bracho-Amador, 2012), determinando incluso en el estudiante diversión, interés y persistencia (Ryan \& Deci, 2000).

Recientemente se ha demostrado que la motivación intrínseca y extrínseca predicen la satisfacción y diversión en la asignatura de EF, relacionando el aburrimiento de forma negativa con la amotivación (Baena-Extremera et al., 2016). De esta forma estos autores afirman que la razón por la cual los estudiantes están satisfechos con la EF se debe probablemente a los altos niveles de motivación, sobre todo la motivación intrínseca. Las creencias de éxito que tenga los estudiantes en EF también influyen en su motivación, según la Teoría de la Atribución de Weiner (1986). Según este constructo las creencias de éxito se dan en función de contextos de logro tras un resultado obtenido por el estudiante, que lo podrá considerar como éxito o fracaso produciéndole una sensación positiva o negativa (Navas, Holgado, Soriano, \& Sampascual, 2008). El estudiante puede adjudicarse el éxito de un resultado a su esfuerzo, la dificultad que conlleva la tarea y al factor suerte; siendo la importancia que atribuida al éxito o al fracaso académico uno de los factores más importantes según Weiner (1986). Sin embargo son escasos los estudios que se han llevado a cabo en el campo de la EF.

Teniendo en cuenta el conocimiento ya existente en este ámbito y en esta línea (Ruíz-Juan, Ortiz-Camacho, García-Montes, Baena-Extremera, \& Baños, 2018), el objetivo de esta investigación fue conocer cómo la satisfacción, la 
motivación y las creencias de éxito en las actividades deportivas de tiempo libre del alumnado de educación secundaria, pueden predecir el clima motivacional percibido en EF en tres contextos culturales diferentes, como son Costa Rica, México y España.

\section{MÉTODO}

\section{Participantes}

Incluidos en este estudio longitudinal, participó un total de 2168 estudiantes del primer curso de Enseñanza Secundaria Obligatoria, provenientes de Costa Rica (423), México (408) y España (1337). Según el género, fueron 1052 chicos (50.4\%) y 1037 chicas (49.6\%), 79 alumnos no reflejando su sexo. Según el tipo de centro educativo, los estudiantes provinieron de centros públicos (86.6\%) y concertados (13.4\%). Según la edad, el rango se ubicó entre 11 y 16 años $(M=12.49 ; D T=.81)$, correspondiéndose la edad media en chicos con 12.53 (DT=.87) años y 12.44 $(D T=.74)$ años en chicas. El estudio longitudinal se efectuó entre febrero-junio de 2011.

\section{Procedimiento}

Primeramente, se solicitó permiso para la realización del trabajo a los centros educativos mediante una carta explicativa de los objetivos de investigación, los procedimientos y adjuntando un modelo del instrumento.

El mismo fue de carácter autoadministrado de aplicación masiva, a completar de forma anónima durante una jornada escolar, previo consenso informado de progenitores y/o tutores, e instrucción de evaluadores. Los participantes así seleccionados recibieron información sobre el objetivo del estudio, su carácter voluntario, de absoluta confidencialidad de las respuestas y manejo de datos. Se les indicó que el instrumento no cotejaba respuestas correctas o incorrectas, por ende, solicitando la mayor posible sinceridad y honestidad.

El estudio posee un informe favorable de la Comisión de Bioética de la Universidad de Murcia.

\section{Instrumentos}

Clima motivacional percibido del profesor de Educación Física de Ruiz-Juan (2014), versión en español del Perceptions of Teacher's Emphasis on Goals Questionnaire (PTEGQ) de Papaioannou, Milosis, Kosmidou, y Tsigilis (2007). Este instrumento fue diseñado para cuantificar las percepciones de los alumnos sobre sus profesores de EF. Está compuesto por 24 ítems y un escalamiento de tipo Likert de cinco puntos (desde 1 -totalmente en desacuerdo- hasta 5 -totalmente de acuerdo). El instrumento consta de cuatro subescalas, a saber: maestría, rendimiento-aproximación, rendimiento-evitación y aprobación social. 
Cuestionario de Satisfacción Intrínseca en el Deporte de Balaguer, Atienza, Castillo, Moreno, y Duda (1997), versión en español del Sport Satisfaction Instrument (SSI) de Duda y Nicholls, (1992). Este instrumento fue diseñado para cuantificar el grado de divertimento al realizar actividades físico-deportivas durante el tiempo libre. Está compuesto de 8 ítems y un escalamiento de tipo Likert de cinco puntos (desde 1 -muy en desacuerdo- a 5 -muy de acuerdo). El instrumento consta de dos escalas: una mide la diversión (5 ítems) y otra, el aburrimiento ( 3 ítems) en la práctica físico-deportiva.

Escala de Motivación Deportiva de Carratalá (2003), versión española de la Sport Motivation Scale (SMS) de Pelletier, Vallerand, Green-Demers, Brière, y Blais (1995). Este instrumento fue diseñado para cuantificar los tres tipos de motivación intrínseca (conocimiento, logro y experiencias estimulantes), así como las tres regulaciones de la motivación extrínseca (externa, introyectada e identificada) y la amotivación. Está compuesto por 28 ítems y un escalamiento de tipo Likert de siete puntos (desde 1 -No se corresponde en absoluto- hasta 7 -Se corresponde totalmente-, con una puntuación intermedia de 4 -Se corresponde medianamente). El instrumento consta de siete subescalas, las cuales se corresponden con los tipos de motivación intrínseca, extrínseca y amotivación mencionados (4 ítems por subescala).

Inventario de Percepción de las Creencias sobre las Causas del Éxito en el Deporte de Castillo, Balaguer y Duda (2002), versión española del Beliefs About the Causes of Sport Success Questionnaire (BACSSQ) de Duda y Nicholls (1992). Este instrumento fue diseñado para cuantificar las percepciones de los sujetos sobre si el esfuerzo (esfuerzo ejercido en el desempeño de la tarea), la habilidad (factores relacionados con la posesión de habilidad) y el uso de técnicas de engaño (uso de conductas engañosas) dan lugar al éxito en el deporte. Está compuesto por 18 ítems (9 para el esfuerzo, 4 para la habilidad y 5 para las técnicas de engaño) y un escalamiento de tipo Likert de cinco puntos (desde 1 -muy en desacuerdo- hasta 5 -muy de acuerdo).

\section{Propiedades psicométricas de los instrumentos}

Para calcular las propiedades psicométricas de las escalas de los cuatro instrumentos se siguió el procedimiento establecido por Carretero-Dios y Pérez (2005). Consecuentemente, no se eliminaron ningún ítem ni las opciones de respuesta en tanto el cumplimiento de los requisitos determinados, según coeficiente de correlación corregido ítem-total $\geq .30$ y desviación típica>1.

Por su parte, el análisis de homogeneidad indicó la ausencia de solapamientos de ítems entre dimensiones teóricas en los cuestionarios. Se halló semejanza con curva normal univariada a partir de los índices de asimetría y curtosis, dado que, según indican Bollen y Long (1994), tales índices se obtuvieron próximos a cero (0) y $<2.0$. 
Para evaluar la validez factorial de las cuatro escalas se utilizó el análisis factorial confirmatorio (AFC). Fueron aspectos procedurales del mismo: el "bootstrapping", el procedimiento de máxima verosimilitud (este último justificándose ante la falta de normalidad multivariada en la mayoría de los datos) y ajuste del modelo con combinación de índices de ajuste absolutos y relativos.

Los modelos de las cuatro escalas presentaron valores adecuados (aceptable bondad de ajuste del modelo original) (Kline, 1998) (Tabla 1). Los coeficientes estandarizados de relación de la variable latente con cada uno de los ítems oscilaron entre .64 y .95. Las cargas factoriales estandarizadas fueron $>60$ y el t-value $>1.96$ en todos los casos, así garantizando la validez convergente de cada instrumento/ escala (Hair, Black, Babin, \& Anderson, 2009). Por su parte, en relación a las subescalas, todas se asociaron con una consistencia interna satisfactoria $(\alpha=.70-.92)$.

Tabla 1. Índices de ajuste del modelo.

\begin{tabular}{|c|c|c|c|c|c|c|c|c|}
\hline & & $n$ & $x^{2} / g l$ & $T L I$ & IFI & CFI & RMSEA & SRMR \\
\hline \multirow{4}{*}{$\begin{array}{l}\text { Costa } \\
\text { Rica }\end{array}$} & Clima motivacional (PTEGQ) & 378 & 1.98 & .95 & .96 & .97 & .04 & .03 \\
\hline & $\begin{array}{l}\text { Satisfacción Intrínseca en el } \\
\text { Deporte (SSI) }\end{array}$ & 311 & 2.59 & .93 & .93 & .93 & .04 & .03 \\
\hline & $\begin{array}{l}\text { Motivación en la práctica físico- } \\
\text { deportiva (SMS) }\end{array}$ & 298 & 2.18 & .95 & .94 & .93 & .03 & .02 \\
\hline & $\begin{array}{l}\text { Percepción de las creencias } \\
\text { sobre las causas del éxito en el } \\
\text { deporte (BACSSQ) }\end{array}$ & 313 & 3.12 & .92 & .92 & .91 & .04 & .04 \\
\hline \multirow{4}{*}{ México } & Clima motivacional (PTEGQ) & 389 & 3.76 & .92 & .91 & .91 & .04 & .05 \\
\hline & $\begin{array}{l}\text { Satisfacción Intrínseca en el } \\
\text { Deporte (SSI) }\end{array}$ & 137 & 3.75 & .94 & .92 & .93 & .06 & .03 \\
\hline & $\begin{array}{l}\text { Motivación en la práctica físico- } \\
\text { deportiva (SMS) }\end{array}$ & 137 & 3.57 & .92 & .91 & .92 & .06 & .04 \\
\hline & $\begin{array}{l}\text { Percepción de las creencias } \\
\text { sobre las causas del éxito en el } \\
\text { deporte (BACSSQ) }\end{array}$ & 394 & 3.43 & .95 & .93 & .95 & .06 & .03 \\
\hline \multirow{5}{*}{ España } & Clima motivacional (PTEGQ) & 1085 & 2.79 & .95 & .95 & .94 & .04 & .04 \\
\hline & $\begin{array}{l}\text { Satisfacción Intrínseca en el } \\
\text { Deporte (SSI) }\end{array}$ & 812 & 3.72 & .95 & .94 & .95 & .06 & .03 \\
\hline & $\begin{array}{l}\text { Motivación en la práctica físico- } \\
\text { deportiva (SMS) }\end{array}$ & 798 & 3.17 & .94 & .94 & .94 & .06 & .04 \\
\hline & $\begin{array}{l}\text { Percepción de las creencias } \\
\text { sobre las causas del éxito en el } \\
\text { deporte (BACSSQ) }\end{array}$ & 1061 & 3.11 & .95 & .93 & .95 & .06 & .03 \\
\hline & \multicolumn{2}{|c|}{ Deseable } & $<5$ & $>.9$ & $>.9$ & $>.9$ & $<.08$ & $<.05$ \\
\hline
\end{tabular}

Fuente: elaboración propia

\section{Análisis de los datos}

Se utilizó el programa SPSS versión 17.0 para Windows para realizar los análisis de ítems, homogeneidad, correlación entre las subescalas (coeficiente de 
Pearson), consistencia interna (alfa de Cronbach), diferencias de medias por países (ANOVA), correlaciones entre todas las dimensiones de las subescalas y regresión lineal jerárquica. Por su parte, se utilizó el programa AMOS versión 21.0 para Windows para examinar la estructura factorial con AFC.

\section{RESULTADOS}

\section{Estadística descriptiva}

Se hallaron diferencias estadísticamente significativas $(p<.001)$ entre las medias de cada variable analizada por países. Con referencia al clima motivacional, las mayores puntuaciones se dan en el clima de maestría $(M=4.05, D T=.73$, México), mientras que las más bajas, en rendimiento-evitación ( $M=2.71, D T=.89$, España). No obstante, el alumnado costarricense obtuvo las puntuaciones más altas en las cuatro subescalas (salvo en maestría), seguido de los alumnados mexicano y español.

Con relación a la satisfacción intrínseca, se hallaron medias altas en la variable diversión en los tres países, el alumnado español y mexicano $(M=4.24$, $D T=.82, M=4.23, D T=.85$, respectivamente) encontrándose ligeramente por encima del alumnado costarricense $(M=3.95, D T=1.00)$. Sin embargo, en la subescala aburrimiento los valores medios fueron bajos, el alumnado español presentando el menor valor de media $(M=1.93, D T=.89)$.

En cuanto a la motivación, el alumnado mexicano presentó valores medios por encima de aquellos de los alumnados costarricense y español en cada una de las variables. Se identificó una tendencia de incremento considerable de las medias a medida que aumentaba el nivel de autodeterminación, pasando en los mexicanos de valores de $M=4.02$ ( $D T=1.63$ ) en amotivación a $M=5.63$ ( $D T=1.28)$ en motivación intrínseca de conocimiento. El alumnado español fue el que presentó una menor media de amotivación $(M=2.83, D T=1.60)$ con diferencias significativas al comparar con el alumnado costarricense $(M=3.57, D T=1.70)$.

En la percepción de las creencias sobre las causas del éxito en el deporte solo se encontraron diferencias estadísticamente significativas $(p<.010)$ en la variable engaño, el alumnado costarricense presentando los valores más elevados $(M=2.84, D T=1.05)$, seguidos de aquellos de los alumnados mexicano $(M=2.73$, $D T=.99)$ y español $(M=2.63, D T=1.00)$, pero siempre con valores inferiores a la creencia de la habilidad (medias 3.17-3.31) y del esfuerzo (medias 3.86-3.96). 
Tabla 2. Coeficiente Alfa, media ( $M$ ) y desviación típica (DT) para Clima motivacional (PTEGQ), Satisfacción intrínseca en el deporte (SSI), Motivación en la práctica físico-deportiva (SMS) y Percepción de las creencias sobre las causas del éxito en el deporte (BACSSQ). Diferencias por países.

\begin{tabular}{|c|c|c|c|c|c|c|c|c|c|c|c|}
\hline & \multicolumn{3}{|c|}{ Costa Rica } & \multicolumn{3}{|c|}{ México } & \multicolumn{3}{|c|}{ España } & \multirow[t]{2}{*}{$=$} & \multirow{2}{*}{ Sig. } \\
\hline & $\alpha$ & $M$ & $D T$ & $\alpha$ & $M$ & $D T$ & $\alpha$ & $M$ & $D T$ & & \\
\hline \multicolumn{12}{|l|}{ PTEGQ } \\
\hline Maestría & .79 & 3.88 & .86 & .73 & 4.05 & .73 & .79 & 3.62 & .87 & 42.45 & .000 \\
\hline $\begin{array}{l}\text { Rendimiento- } \\
\text { aproximación }\end{array}$ & .78 & 3.24 & 1.01 & .76 & 3.16 & .97 & .78 & 2.76 & .96 & 46.51 & .000 \\
\hline Rendimiento-evitación & .79 & 3.02 & 1.05 & .73 & 2.85 & .96 & .72 & 2.71 & .89 & 15.75 & .000 \\
\hline Aprobación social & .85 & 3.40 & 1.07 & .85 & 3.36 & 1.05 & .85 & 3.01 & 1.01 & 28.75 & .000 \\
\hline \multicolumn{12}{|l|}{ SSI } \\
\hline Diversión & .84 & 3.95 & 1.00 & .80 & 4.23 & .85 & .85 & 4.24 & .82 & 12.39 & .000 \\
\hline Aburrimiento & .70 & 2.28 & 1.12 & .76 & 2.00 & 1.07 & .73 & 1.93 & .89 & 14.60 & .000 \\
\hline \multicolumn{12}{|l|}{ SMS } \\
\hline Motivación Intrínseca & .91 & 4.70 & 1.42 & .87 & 5.44 & 1.14 & .92 & 5.06 & 1.33 & 15.62 & .000 \\
\hline Conocimiento & .79 & 4.84 & 1.56 & .76 & 5.63 & 1.28 & .80 & 5.16 & 1.43 & 14.02 & .000 \\
\hline Logro & .78 & 4.76 & 1.54 & .72 & 5.48 & 1.27 & .77 & 5.11 & 1.39 & 13.11 & .000 \\
\hline $\begin{array}{l}\text { Experiencias } \\
\text { estimulantes }\end{array}$ & .79 & 4.51 & 1.58 & .74 & 5.23 & 1.32 & .81 & 4.96 & 1.48 & 13.87 & .000 \\
\hline Motivación Extrínseca & .89 & 4.50 & 1.32 & .86 & 5.26 & 1.15 & .87 & 4.60 & 1.27 & 18.64 & .000 \\
\hline Externa & .75 & 4.22 & 1.53 & .77 & 5.04 & 1.40 & .74 & 4.20 & 1.55 & 18.09 & .000 \\
\hline Introyectada & .77 & 4.59 & 1.37 & .74 & 5.44 & 1.25 & .77 & 4.92 & 1.36 & 18.70 & .000 \\
\hline Identificada & .79 & 4.70 & 1.55 & .74 & 5.31 & 1.28 & .71 & 4.68 & 1.43 & 11.20 & .000 \\
\hline Amotivación & .76 & 3.57 & 1.70 & .76 & 4.02 & 1.63 & .74 & 2.83 & 1.60 & 44.44 & .000 \\
\hline \multicolumn{12}{|l|}{ BACSSQ } \\
\hline Esfuerzo & .87 & 3.86 & .89 & .86 & 3.95 & .83 & .87 & 3.96 & .82 & 1.83 & .160 \\
\hline Habilidad & .77 & 3.17 & 1.01 & .74 & 3.31 & .94 & .70 & 3.26 & .92 & 1.74 & .175 \\
\hline Engaño & .78 & 2.84 & 1.05 & .74 & 2.73 & .99 & .79 & 2.63 & 1.00 & 5.39 & .005 \\
\hline
\end{tabular}

Fuente: elaboración propia

Relaciones del clima motivacional con la satisfacción intrínseca, motivación y creencias de las causas del éxito en el deporte

En la Tabla 3 quedan reflejados los resultados de las correlaciones calculadas, con resultados muy similares en los tres países. Con respecto a la correlación de los factores del clima motivacional en EF en los tres países, todos se intercorrelacionaron positiva y significativamente.

Por otro lado, la maestría correlaciona baja y moderadamente de manera positiva con el resto de variables, salvo con aburrimiento, amotivación, habilidad y engaño. Del mismo modo, el rendimiento-aproximación y el rendimiento-evitación, correlacionan baja y moderadamente de manera positiva con aburrimiento, motivación extrínseca, regulación externa (salvo en rendimiento-evitación), regulación introyectada, regulación identificada, amotivación, habilidad y engaño, y negativamente con esfuerzo, no correlacionando con el resto de variables. Por 
Rev.int.med.cienc.act.fís.deporte - vol. 19 - número 74 - ISSN: 1577-0354

último, la aprobación social correlaciona positiva, baja y moderadamente con el resto de variables, salvo con diversión, aburrimiento y esfuerzo. 
Tabla 3. Correlaciones entre las subescalas del Clima motivacional (PTEGQ), Satisfacción intrínseca en el deporte (SSI), Motivación en la práctica físico-deportiva (SMS) y Percepción de las creencias sobre las causas del éxito en el deporte (BACSSQ). Diferencias por países.

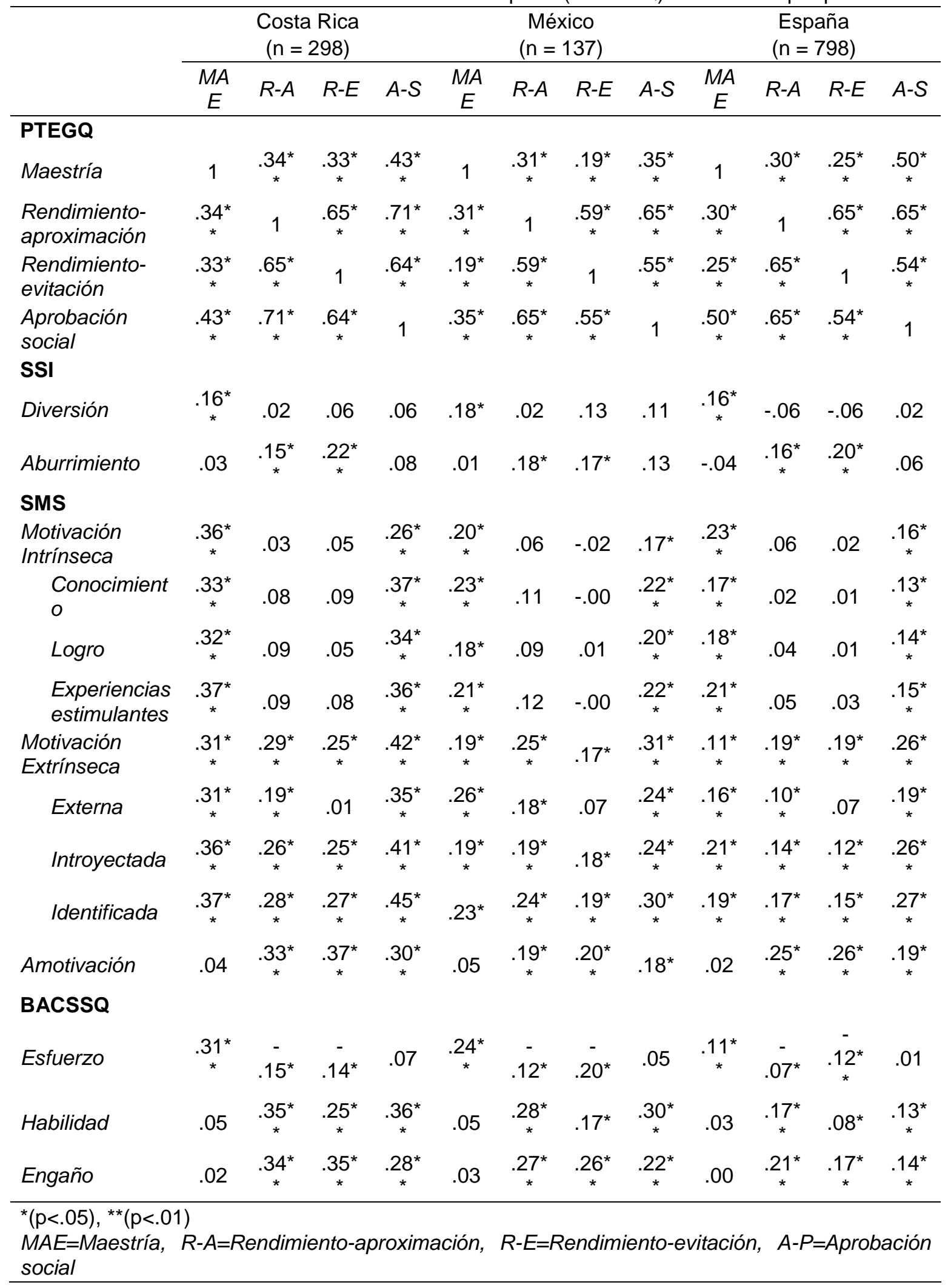


Fuente: elaboración propia

\section{Análisis regresivo multivariante}

En este análisis se incluyeron sólo los sujetos activos; esto es, aquellos que realizan actividad físico-deportiva en su tiempo libre. Se realizó un análisis de regresión lineal multivariado tomando como variable dependiente la media del clima motivacional (y sus subescalas) y como predictora, cada una de las variables de Satisfacción intrínseca en el deporte, Motivación en la práctica físico-deportiva y Percepción de las creencias sobre las causas del éxito en el deporte. Como variable de selección se utilizó el país.

Se obtuvieron unos sólidos modelos que explicaron una gran parte de la varianza en cada país (33\%-49\%). Se extrajeron los valores de $R^{2}$, de Beta y el de $F$ para explicar la varianza, la predicción entre variables y la existencia de relaciones entre las variables seleccionadas y su significatividad, respectivamente (Tabla 4).

Los modelos muestran que la maestría, en los tres países, se puede predecir significativamente por puntuar alto en diversión, motivación intrínseca, motivación extrínseca (salvo en México) y esfuerzo ( $40 \%$ de varianza en Costa Rica, $41 \%$ en México y $35 \%$ en España).

Por otra parte, el rendimiento-aproximación, en los tres países, se puede predecir significativamente por una alta puntuación en motivación extrínseca, amotivación y habilidad y por puntuar bajo en diversión y esfuerzo. En México, se predice, además, por una puntuación alta en aburrimiento ( $43 \%$ de varianza en Costa Rica, 38\% en México y 34\% en España).

Los modelos de rendimiento-evitación, en los tres países, se pueden predecir significativamente por una alta puntuación en aburrimiento, motivación extrínseca (salvo en Costa Rica) y amotivación y por puntuar bajo en esfuerzo ( $43 \%$ de varianza en Costa Rica, 38\% en México y 33\% en España).

Por último, los modelos de aprobación social, en los tres países, se pueden predecir significativamente por una alta puntuación en motivación extrínseca y habilidad (salvo en España) (48\% de varianza en Costa Rica, 45\% en México y 34\% en España).

Tabla 4. Análisis Regresivo Lineal Multivariado: modelos que predicen significativamente el Clima motivacional (PTEGQ) en función de Satisfacción intrínseca en el deporte (SSI), Motivación en la práctica físico-deportiva (SMS) y Percepción de las creencias sobre las causas del éxito en el deporte (BACSSQ). 
Rev.int.med.cienc.act.fís.deporte - vol. 19 - número 74 - ISSN: 1577-0354

\begin{tabular}{|c|c|c|c|c|c|c|c|c|c|c|c|c|}
\hline & \multicolumn{4}{|c|}{$\begin{array}{c}\text { Costa Rica } \\
(\mathrm{n}=298)\end{array}$} & \multicolumn{4}{|c|}{$\begin{array}{c}\text { México } \\
(\mathrm{n}=137)\end{array}$} & \multicolumn{4}{|c|}{$\begin{array}{c}\text { España } \\
(\mathrm{n}=798)\end{array}$} \\
\hline & $M A E$ & $R-A$ & $R-E$ & $A-S$ & $M A E$ & $R-A$ & $R-E$ & $A-S$ & $M A E$ & $R-A$ & $R-E$ & $A-S$ \\
\hline & $\begin{array}{l}\mathrm{Bn}_{\mathrm{gn}} \\
\mathrm{Bi}\end{array}$ & $\underset{\mathrm{gn}}{\mathrm{BetaSi}}$ & $\begin{array}{c}\text { BetaSi } \\
\mathrm{gn}\end{array}$ & $\begin{array}{l}\mathrm{Beta}_{\mathrm{gn}} \\
\end{array}$ & $\begin{array}{l}\text { BetaSi } \\
\text { gn }\end{array}$ & $\underset{\mathrm{gn}}{\mathrm{Beta}}$ & $\begin{array}{c}\text { BetaSi } \\
\text { gn }\end{array}$ & $\begin{array}{c}\text { Beta } \\
\text { gn }\end{array}$ & $\begin{array}{l}\mathrm{Beta}_{\mathrm{gn}}^{\mathrm{Si}} \\
\end{array}$ & $\underset{\mathrm{gn}}{\mathrm{Beta}^{\mathrm{Si}}}$ & $\begin{array}{l}\mathrm{Beta}_{\mathrm{gn}} \\
\end{array}$ & $\underset{\mathrm{gn}}{\mathrm{Beta}}$ \\
\hline \multicolumn{13}{|l|}{ SSI } \\
\hline Diversión & $.20^{\star *}$ & $-.10^{*}$ & -.04 & -.05 & $.24^{*}$ & $-.20^{\star}$ & .08 & .06 & $.10^{*}$ & $-.06^{*}$ & -.02 & -.02 \\
\hline $\begin{array}{l}\text { Aburrimie } \\
\text { nto }\end{array}$ & -.04 & -.02 & $.18^{*}$ & -.01 & .06 & $.20^{*}$ & $.21^{*}$ & .13 & .04 & .06 & $.09^{*}$ & .00 \\
\hline \multicolumn{13}{|l|}{ SMS } \\
\hline $\begin{array}{l}\text { Motivació } \\
n \\
\text { Intrínseca }\end{array}$ & $.15^{\star}$ & -.01 & .12 & .09 & $.21^{*}$ & -.03 & -.15 & -.07 & $.10^{*}$ & -.03 & -.05 & -.05 \\
\hline $\begin{array}{l}\text { Motivació } \\
n \\
\text { Extrínsec } \\
\text { a }\end{array}$ & $.16^{*}$ & $.16^{*}$ & .09 & $.30^{\star *}$ & .10 & $.20^{*}$ & $.23^{*}$ & $.26^{* *}$ & $.10^{*}$ & $.16^{\star *}$ & $.17^{\star *}$ & $.30^{* * *}$ \\
\hline $\begin{array}{l}\text { Amotivaci } \\
\text { ón }\end{array}$ & .02 & $.20^{\star \star}$ & $.25^{\star * *}$ & .10 & .04 & $.24^{*}$ & $.25^{\star \star}$ & .05 & .00 & $.11^{*}$ & $.13^{\star *}$ & .06 \\
\hline \multicolumn{13}{|l|}{ BACSSQ } \\
\hline Esfuerzo & $.19^{*}$ & $-.11^{*}$ & $-.11^{*}$ & -.08 & $.36^{* *}$ & $-.22^{*}$ & $-.21^{*}$ & .09 & $.09^{*}$ & $-.12^{\star \star}$ & $-.10^{*}$ & -.01 \\
\hline Habilidad & .09 & $.19^{*}$ & .03 & $.18^{*}$ & -.05 & $.33^{* *}$ & .17 & $.30^{\star *}$ & -.06 & $.13^{\star *}$ & .03 & .00 \\
\hline Engaño & -.00 & .10 & .08 & -.00 & .10 & .11 & .14 & -.09 & -.00 & .05 & .04 & .08 \\
\hline \multicolumn{13}{|c|}{ 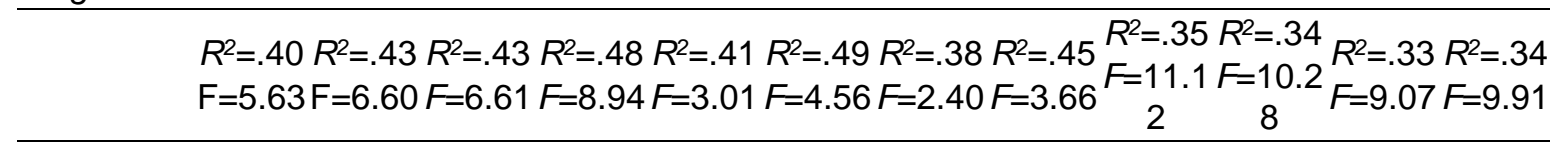 } \\
\hline $\begin{array}{l}\text { * }(\mathrm{p}<.05),{ }^{*} \\
\text { MAE }=\text { Mae } \\
\text { social }\end{array}$ & $\begin{array}{l}(\mathrm{p}<.01) \\
\text { stría, }\end{array}$ & $\begin{array}{l}{ }^{* * *}(\mathrm{p}< \\
3-A=R\end{array}$ & $\begin{array}{l}.001) \\
\text { ndimier }\end{array}$ & on & 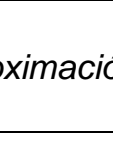 & & $=n e$ & 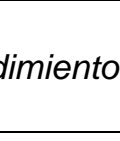 & & & $P=A$ & acic \\
\hline
\end{tabular}

Fuente: elaboración propia

\section{DISCUSIÓN}

Como expresan Alcaraz-Ibáñez, (2017) Gallegos, Ruiz-Juan, Villareal, y Zamarripa (2019), durante la adolescencia el ser humano experimenta una serie de profundas transformaciones que, en algunos casos, pueden dar lugar a la aparición de muchos tipos de conductas diversas. Teniendo en cuenta esto, analizar una muestra de sujetos importante de tres países, podría arrojar datos esclarecedores. Por este motivo, el objetivo de este estudio fue conocer como la satisfacción, la motivación y las creencias de éxito en las actividades deportivas de tiempo libre del alumnado de educación secundaria, pueden predecir el clima motivacional percibido en EF en tres contextos culturales diferentes, como son Costa Rica, México y España. Variables como la motivación, satisfacción intrínseca y las creencias de éxito en las actividades físico-deportivas en el tiempo libre pueden predecir el clima motivacional en el aula de EF.

En este estudio se obtuvo la mayor puntuación se dan en el clima a la maestría y las más bajas en rendimiento-evitación. Estos resultados son similares en los estudiantes de los tres países estudiados, lo que es seguro afirmar que esta 
es la tendencia general de los estudiantes de EF analizados (2168 adolescentes) y que está en la línea con los trabajos realizados por Méndez-Giménez, FernándezRío, Cecchini, y González. (2013) y otros trabajos de Ruiz-Juan et al. (2018). Por otro lado, es de destacar que los valores más altos en maestría fueron obtenidos por estudiantes mexicanos, mientras que los estudiantes españoles las puntuaciones más bajas en evitación-rendimiento. Igualmente, en las cuatro subescalas, el alumnado costarricense (salvo en maestría) obtiene las puntuaciones más altas, seguido del mexicano y del español.

En cuanto a la satisfacción intrínseca en las actividades físico-deportivas en el tiempo libre, la variable diversión obtuvo la mayor puntuación en los tres países estando el alumnado costarricense ligeramente por debajo del alumnado español y mexicano. La variable aburrimiento por el contrario, presentó los valores más bajos siendo los españoles quienes menos amotivados y aburridos están. Reforzando esta idea, otros trabajos confirman que los alumnos sentían mayor diversión que aburrimiento en las actividades físico-deportivas en el tiempo libre (Abraldes, Gómez-López, Granero-Gallegos, \& Rodríguez-Suárez, 2013). También en el área de la EF se han obtenido las puntuaciones más bajas en aburrimiento (MéndezGiménez et al., 2013). Estos datos son de gran relevancia, puesto que se observa que los tres países siguen la misma tendencia, estando demostrado que la satisfacción en EF predice positivamente la satisfacción en la escuela (BaenaExtremera \& Granero-Gallegos, 2015).

Respecto a la motivación, la tendencia general en los tres países es obtener altas puntuaciones de motivación intrínseca y menores puntuaciones en la desmotivación, obteniendo los estudiantes mexicanos los valores medios más altos y los españoles son los que presentan menor amotivación. Estos datos están en línea con algunos estudios recientes que muestran altas puntuaciones medias en autodeterminación y puntuaciones más bajas en la desmotivación (Moreno-Murcia, Zomeño, Marín, Ruiz, \& Cervelló, 2013). Lim y Wang (2009) mostraron que los estudiantes con mayores niveles de motivación intrínseca, tienen mayores probabilidades de convertirse físicamente activos. De esta forma se puede afirmar que los adolescentes que practican deportes en su tiempo libre, son los que disponen de una motivación más autodeterminada hacia la práctica deportiva, debiendo tener cautela con aquellos que se encuentren desmotivados, puesto que la amotivación puede acarrear el abandono de la práctica deportiva en el tiempo libre.

En la percepción de las creencias sobre las causas del éxito en el deporte, la habilidad y el esfuerzo fueron la causas más destacadas por los estudiantes de los tres países, destacando la variable engaño sobre todo en los estudiantes costarricenses, seguidos de mexicanos y españoles respectivamente. Resultados similares obtuvieron otras investigaciones (Castillo et al., 2002; Navas et al., 2008) siendo el esfuerzo y la habilidad la prevalencia entre los jóvenes deportistas por encima de las técnicas de engaño. 
En cuanto al análisis predictivo, los resultados muestran que un clima de aproximación y evitación al rendimiento se relacionan positivamente con el aburrimiento, motivación extrínseca, regulación introyectada, regulación identificada. Resultados parecidos obtuvieron Smith et al. (2002) relacionando el clima de rendimiento con la motivación extrínseca y metas de rendimiento. En este estudio también se correlaciona un clima de aproximación y evitación del rendimiento con la amotivación del estudiante, siguiendo la línea de Conroy, Kaye y Coatsworth (2006) que además relacionan los climas de rendimiento con la desmotivación, ansiedad y un aprendizaje desadaptativo. Sin embargo, la variable regulación externa solo se relaciona en este estudio con un clima de aproximación al rendimiento, sin existir relación con la evitación del rendimiento encontrando similitudes con otros estudios (Gutiérrez, 2014; Vílchez \& Ruiz-Juan, 2016).

Ambos climas orientados al rendimiento tienen relación con técnicas de engaño en el deporte y de demostración de habilidad, lo cual es lógico, puesto que un clima de aproximación al rendimiento busca que el estudiante demuestre su habilidad por encima de sus iguales, y un clima de evitación-rendimiento pretende evitar hacerlo peor que sus compañeros (Elliot \& McGregor, 2001). Por el contrario el esfuerzo se correlaciona de forma negativa con climas de rendimiento, ya que los adolescentes consideran la habilidad como algo permanente y dependiente del talento propio, no creyendo en el esfuerzo.

Destacar que todos los estudiantes de los tres países obtuvieron mayor percepción de un clima de maestría o aprendizaje en las clases de EF al igual que en otros estudios anteriormente realizados (Granero-Gallegos \& Baena-Extremera, 2014; Soini et al., 2014). Variables como la diversión, la motivación intrínseca, extrínseca y el esfuerzo en las actividades físico-deportivas de tiempo libre, predicen de forma positiva un clima motivacional orientado a la maestría. Baena-Extremera et al. (2015) relacionaron que un clima de maestría se relacionaba con mayor satisfacción y diversión en las clases de EF, así como mayor motivación intrínseca.

La motivación extrínseca, intrínseca, demostración de habilidad (salvo en España) y técnicas de engaño en las actividades físico-deportivas en el tiempo libre predicen en este estudio un clima motivacional de aprobación social. Los adolescentes adquieren un comportamiento el cual se trata de la búsqueda de la demostración de superioridad respecto a sus compañeros con el objetivo de obtener reconocimiento social, independientemente de los resultados de la ejecución (Papaioannou et al., 2007).

Lo novedoso que aporta este estudio, es que a partir de la motivación, satisfacción y creencias de éxito que presente el adolescente en sus actividades deportivas en su tiempo libre se puede predecir el clima motivacional creado por el docente en EF. De esta forma se relaciona por un lado el rendimiento-aproximación, en los tres países, se puede predecir significativamente por una alta puntuación en motivación extrínseca, amotivación y habilidad y por puntuar bajo en diversión y esfuerzo. En México, se predice, además, por una puntuación alta en aburrimiento. De otra parte los modelos de rendimiento-evitación, en los tres países, se pueden 
predecir significativamente por una alta puntuación en aburrimiento, motivación extrínseca (salvo en Costa Rica) y amotivación y por puntuar bajo en esfuerzo. Sin embargo Bortoli et al. (2015) consideran un clima de maestría como el más adecuado para aumentar la motivación de los escolares. En este estudio, los modelos muestran que la maestría, en los tres países, se puede predecir significativamente por puntuar alto en diversión, motivación intrínseca, motivación extrínseca (salvo en México) y esfuerzo. Así, cabe destacar la importancia de que los alumnos se encuentren satisfechos y motivados en estas edades puesto que es donde mayor tasa de abandono deportivo se da, y es una etapa de la vida crucial para la construcción de un estilo de vida saludable (Nuviala, Tamayo, \& Nuviala, 2012). Ewing (1981) llegó a la conclusión de que los estudiantes que abandonan la práctica de actividad física están más orientados al ego, mientras que los que más participan en actividad física están orientados a la aprobación social.

Como conclusión, la contribución de este estudio a la literatura especializada debe destacarse, puesto que muestra las tendencias generales a través de una muestra de los tres países. La satisfacción, motivación y creencias de éxito en el deporte pueden predecir el clima motivacional en el aula de EF, aportando pistas sobre cómo organizar y dirigir las clases de EF, siendo aconsejable los climas de aproximación a la maestría por encima de los climas de evitación y aproximación del rendimiento (Elliot \& McGregor, 2001); consiguiendo mayores intenciones de continuar en la práctica físico-deportiva en el tiempo libre (Moreno-Murcia, Huéscar, \& Cervelló, 2012). 


\section{REFERENCIAS BIBLIOGRÁFICAS}

Abraldes, J. A., Gómez-López, M., Granero-Gallegos, A., \& Rodríguez-Suárez, N. (2013). The goal orientation of the lifesavers and the relationship with the satisfaction and the beliefs about the causes of success in sport. CCD. Cultura, Ciencia,Deporte, $8(22)$,

$59-66$. https://doi.org/10.12800/ccd.v8i22.230

Alcaraz-Ibáñez, M. (2017). Comparación social de la apariencia en contextos de ejercicio físico como variable predictora de los trastornos de la conducta alimentaria en adolescentes de ambos sexos. Espiral. Cuadernos del Profesorado, 10(21), 80-89. http://dx.doi.org/10.25115/ecp.v10i21.

Baena-Extremera, A., Gómez-López, M., Granero-Gallegos A., \& Ortiz-Camacho, M. (2015). Predicting Satisfaction in Physical Education from Motivational Climate and Self-determined Motivation. Journal of Teaching in Physical Education, 34, 210-224. https://doi.org/10.1123/itpe.2013-0165

Baena-Extremera, A., \& Granero-Gallegos, A. (2015). Modelo de predicción de la satisfacción con la educación física y la escuela. Revista de Psicodidáctica, 20(1), 177-192. https://doi.org/10.1387/RevPsicodidact.11268

Baena-Extremera, A., Granero-Gallegos, A., Ponce-de-León-Elizondo, A., SanzArazuri, E., Valdemoros-San-Emeterio, M. D. L., \& Martínez-Molina, M. (2016). Psychological factors related to physical education classes as predictors of students' intention to partake in leisure-time physical activity. Ciência \& Saúde Coletiva, 21(4), 1105-1112. https://doi.org/10.1590/141381232015214.07742015

Balaguer, I., Atienza, F. L., Castillo, I., Moreno, Y., \& Duda, J. L. (1997). Factorial structure of measures of satisfaction/interest in sport and classroom in the case of Spanish adolescents. Fourth European Conference of Psychological Assessment. Lisboa, Portugal.

Baños, R., Ortiz-Camacho, M. M., Baena-Extremera, A., \& Tristán-Rodríguez, J. L. (2017). Satisfacción, motivación y rendimiento académico en estudiantes de secundaria y bachillerato: antecedentes, diseño, metodología y propuesta de análisis para un trabajo de investigación. Espiral: Cuadernos del Profesorado, 10(20), 40-50. http://dx.doi.org/10.2511.

Bollen, K. A. \& Long, J. (1994). Testing structural equation models. Newbury Park, CA: Sage.

Carratalá, E. (2003). Análisis de la teoría de las Metas de Logro y de la Autodeterminación en los planes de especialización deportiva de la Generalitat Valenciana. Tesis Doctoral sin publicar, Facultad de Psicología, Universidad de Valencia, Valencia, España.

Carretero-Dios, H. \& Pérez, C. (2005). Normas para el desarrollo y revisión de estudios instrumentales. International Journal of Clinical and Health Psychology, 5, 521-551.

Castillo, I., Balaguer, I., \& Duda, J. L. (2002). Las perspectivas de meta de los adolescentes en el contexto deportivo. Psicothema, 14(2), 280-287. 
Castillo, I., Balaguer, I., Duda, J. L., \& García-Merita, M. L. (2004). Factores psicosociales asociados con la participación deportiva en la adolescencia. Revista latinoamericana de psicología, 36(3), 505-515.

Conroy, D. E., Kaye, M. P., \& Coatsworth, J. D. (2006). Coaching climates and the destructive effects of mastery-avoidance achievement goals on situational motivation. Journal of Sport and Exercise Psychology, 28, 69-92. https://doi.org/10.1123/jsep.28.1.69

Cook, D. A., Castillo, R. M., Gas, B., \& Artino, A. R. (2017). Measuring achievement goal motivation, mindsets and cognitive load: validation of three instruments' scores. Medical Education, 51(10), 1061-1074. https://doi.org/10.1111/medu.13405

Deci, E.L., \& Ryan, R.M. (2000). The "what" and "why" of goal pursuits: Human needs and the self-determination of behaviour. Psychological Inquiry, 11, 227-268. https://doi.org/10.1207/S15327965PLI1104_01

Duda, J. L. \& Nicholls, J. G. (1992). Dimensions of achievement motivation in schoolwork and sport. Journal of Educational Psychology, 84(3), 290-299. https://doi.org/10.1037/0022-0663.84.3.290

Elliot, A. J. \& McGregor, H. A. (2001). A 2x2 achievement goal framework. Journal of Personality and Social Psychology, 80, 501-519. https://doi.org/10.1037//0022-3514.80.3.501

Ewing, M. E. (1981). Achievement orientations and sport behavior of males and females. Unpublished doctoral dissertation, University of Illinois at UrbanaChampaign.

Gallegos, J. J., Ruiz-Juan, F., Villarreal, M. A., \& Zamarripa, J. I. (2019). Etapas de cambio en la práctica física de tiempo libre en estudiantes de secundaria de Victoria de Durango, México. Retos, 35, 196-200.

Gómez-López, M., Granero-Gallegos, A., Baena-Extremera, A., \& Abraldes, A. (2014), Goal orientation effects on elite handball players motivation and motivational climate. Procedia Social and Behavioral Sciences, 132, 434-440. https://doi.org/10.1016/j.sbspro.2014.04.333

Granero-Gallegos, A. \& Baena-Extremera, A. (2014). Prediction of self-determined motivation as goal orientations and motivational climate in Physical Education. Retos, 25, 23-27.

Granero Gallegos, A., Baena Extremera, A., Pérez Quero, F.J., Ortíz Camacho, M. M., \& Bracho Amador, C. (2012). Analysis of motivational profiles of satisfaction and importance of physical education in high school adolescents. Journal of Sports Science \& Medicine, 11, 614-623

Gutiérrez, M. (2014). Relaciones entre el clima motivacional, las experiencias en educación física y la motivación intrínseca de los alumnos. Retos, 26, 9-15

Hair, J. F., Black, W. C., Babin, B. J., \& Anderson, R. E. (2009). Multivariate Data Analysis ( $7^{\text {th }}$ ed.). New York: Pearson Prentice Hall.

Juliá, P., \& Baena-Extremera, A. (2018). Mejora de la motivación y las metas sociales tras la realización de una unidad didáctica de escalada. Espiral: Cuadernos del profesorado, 11(22), 44-51. http://dx.doi.org/10.25115/ecp.v11i21

Kline, R. (1998). Principles and practice of structural equation modeling. New York: 
Guilford.

Lim, B. S. C., \& Wang, C. K. J. (2009). Perceived autonomy support, behavioural regulations in physical education and physical activity intention. Psychology of Sport and Exercise, 10, 52-60. https://doi.org/10.1016/j.psychsport.2008.06.003

Méndez-Giménez, A., Fernández-Río, J., Cecchini, J. A., \& González, C. (2013). Perfiles motivacionales y sus consecuencias en educación física. Un estudio complementario de metas de logro $2 \times 2$ y autodeterminación. Revista de Psicología del Deporte, 22(1), 29-38.

Moreno-Murcia, J. A., Huéscar, E., \& Cervelló, G. (2012). Prediction of adolescents doing physical activity after completing secondary education. Spanish Journal of Psychology, 15(1), 90-100. https://doi.org/10.5209/rev_SJOP.2012.v15.n1.37288

Moreno-Murcia, J. A., Zomeño, T., Marín, L. M., Ruiz, L. M., \& Cervelló, E. (2013). Percepción de la utilidad e importancia de la educación física según la motivación generada por el docente. Revista de Educación, 362, 380-401.

Navas, L., Holgado, F. P., Soriano, J. A., \& Sampascual, G. (2008). El cuestionario de atribuciones para educación física: Análisis exploratorio y confirmatorio. Acción Psicológica, 5(2), 77-85.

Nicholls, J.G. (1989). The competitive ethos and democratic education. Cambridge, MA: Harvard University Press.

Nuviala, A.; Tamayo, J. A., \& Nuviala R. (2012). Perceived quality of the school sport as predictor of sports dropout in teenagers. Revista Internacional de Medicina y Ciencias de la Actividad Física y el Deporte, 12(47), 389-404.

Papaioannou, A., Milosis, D., Kosmidou, E., \& Tsigilis, N. (2007). Motivational climate and achievement goals at the situational level of generality. Journal of Applied Sport Psychology, 19, 38-66. https://doi.org/10.1080/10413200601113778

Pelletier, L. G., Vallerand, R. J., Green-Demers, I., Brière, N. M., \& Blais, M. R. (1995). Leisure and mental health: relationship between leisure involvement and psychological well-being. Canadian Journal of Behavioural Science, 27, 214-225.

Roberts, G. \& Treasure, D. (2018). Advances in Motivation in Sport and Exercise (3rd ed.). Champaign, IL: Human Kinetics.

Ruiz-Juan, F., Ortiz-Camacho, M. M., García-Montes, M. M., Baena-Extremera, A., \& Baños, R. (2018). Predicción transcultural del clima motivacional en educación física. Revista Internacional de Medicina y Ciencias de la Actividad Física y el Deporte vol. 18 (69) pp. 165183. https://doi.org/10.15366/rimcafd2018.69.011

Ryan, R. M. \& Deci, E. L. (2000). Self-determination theory and the facilitation of intrinsic motivation. social development and well-being. American Psychologist, 55(1), 68-78. http://dx.doi.org/10.1037//0003-066X.55.1.68

Smith, M., Duda, J. L., Allen, J., \& Hall, H. K. (2002). Contemporary measures of approach and avoidance goal orientations: Similarities and differences. British Journal of Educational Psychology, 72, 155-190. https://doi.org/10.1348/000709902158838 
Soini, M., Liukkonen, J., Watt, A., Yli-Piipari, S., \& Jaakkola, T. (2014). Factorial Validity and Internal Consistency of the Motivational Climate in Physical Education Scale. Journal of Sports Science and Medicine, 13, 137-144.

Vella, S. A., Swann, C., Allen, M. S., Schweickle, M. J., \& Magee, C. A. (2016). Bidirectional Associations between Sport Involvement and Mental Health in Adolescence. Medicine and Science in Sports and Exercise, 49(4), 687-694. https://doi.org/10.1249/MSS.0000000000001142

Vílchez, M. P. \& Ruiz-Juan, F. (2016). Clima motivacional en Educación Física y actividad físico-deportiva en el tiempo libre en alumnado de España, Costa Rica y México. Retos, 29, 195-200.

Weiner, B. (1986). An attributional theory of achievement motivation and emotion. New York: Springer-Verlag. https://doi.org/10.1007/978-1-4612-4948-1

Número de citas totales / Total references: 43 (100\%)

Número de citas propias de la revista / Journal's own references: 1 (2.32\%)

Rev.int.med.cienc.act.fís.deporte - vol. 19 - número 74 - ISSN: 1577-0354 